\title{
Bone regeneration of decellularized in-vivo deposited extracellular matrix (ECM) on hydroxyapatite sponge scaffold
}

\section{Introduction}

Large bone defects and bone fractures do not heal spontaneously and considered to be one of the most challenging cases in bone reconstruction. For many fractures and bone defects, the use of hardware fixation alone is not enough to ensure fracture healing. Use of autograft, allogenic and xenegeneic scaffolds have some limitations and complications such as donor site pain and limited availability of different sizes and shapes making large defects impractical to treat. ${ }^{1}$ Different approach in tissue engineering were developed and studied to address this problem with the use of hydroxyapatite, a widely used calcium phosphate in biomedical applications, due to its close chemical composition to bones and teeth and has an excellent properties such as biocompatibility and osteoconductivity but lack intrinsic osteogenic potential found in autografts. ${ }^{2}$ The ECM is a complicated complex of many kinds of polysaccharides and proteins, such as collagens, proteoglycans and glycoproteins that are essential in tissue engineering. ${ }^{3}$ Cellular interactions with the ECM are known to play a critical role in providing structural support to cells, directing cell function, and regulating development, homeostasis, and repair of a variety of tissues. ${ }^{4-8}$ ECM derived and ECM mimicking scaffolds have shown promising results in facilitating the constructive remodeling of many different tissues in preclinical and applications. ${ }^{3,9,10}$ To obtain an ECM derived or ECM mimicking scaffolds, decellularization process were employed in native tissues and organs to remove the cellular and nuclear contents while maintaining the important proteins present in the ECM. Studies have been focus on the generation of cell derived ECM for different tissue engineering applications. ${ }^{11-13}$ However, different factors need to be considered in generation of cell derived ECM such as type of cells and the manner in which cell are cultured that involves the use of appropriate media, addition of growth factors, bioreactor design and flow rate. ${ }^{14}$ The aim of this study is to determine the feasibility and effect of in-vivo deposited extracellular matrix on bone regeneration capacity of porous hydroxyapatite sponge scaffold.

In our study, ${ }^{15}$ decellularized in-vivo deposited extracellular matrix could enhance the bone regeneration properties of hydroxyapatite (HA) scaffolds. HA sponge scaffolds were implanted in subcutaneous pockets of rats to generate ECM and extracted after 1, 2 and 3weeks (Figure 1). Decellularization treatment of in-vivo deposited HA scaffolds were employed with the use of $1 \%$ SDS, agitation and sonication to facilitate effective removal of cells. DNA and RNA content of the in-vivo deposited ECM were significantly removed after decellularization with preserved major components. DNA and RNA quantification showed decellularized in-vivo deposited ECM HA scaffolds have met the requirements Crapo et.al reported that decellularized ECM should have a DNA content of $<50 \mathrm{ng} / \mathrm{mg}$ dried ECM to avoid immune reaction. ${ }^{16}$ In addition, several studies reported that DNA component directly correlated with adverse host reaction upon in-vivo implantation or introduction of new cell line. ${ }^{16-20} \mathrm{ECM}$ is composed of wide array of ECM components such as collagen, a major ECM component that provides mechanical strength and
Volume 3 Issue 3 - 2016

\author{
Reiza Ventura,' Andrew Padalhin, ' Young-Ki \\ Min, ${ }^{1,2}$ Byong-Taek Lee ${ }^{1,3}$ \\ 'Department of Regenerative Medicine, Soonchunhyang \\ University, South Korea \\ ${ }^{2}$ Department of Physiology, Soonchunhyang University, South \\ Korea \\ ${ }^{3}$ Institute of Tissue Regeneration, Soonchunhyang University, \\ South Korea
}

Correspondence: Reiza Ventura, Department of Regenerative Medicine, College of Medicine, Soonchunhyang University, South Korea, Email venturareiza@gmail.com

Received: February 23, 2016 | Published: April 28, 2016

promotes cell adhesion, sulfated glycosaminoglycans (sGAG) which bind growth factors, promotes water retention and contributes to the gel properties of ECM and proteoglycans that serve as the integral membrane protein that modulates growth and proliferation of cells as well as growth factors. ${ }^{21,22}$ Retention ECM components while thorough removal of cellular and nuclear components are the goals of decellularization. Decellularization treatment removed $73 \%$ of collagen content in the ECM due to agitation and sonication that lead to significant reduction of mechanical strength, increased in porosity and pore size distribution of all decellularized scaffolds. However, $57 \%$ of sulfated glycosaminoglycan were still present in decellularized ECM as well as glycoproteins as revealed by PAS staining indicating that important ECM components were not thoroughly removed after decellularization (Figure 2). In-vitro studies revealed decellularized scaffolds have no cytotoxic effect in MC3T3-E1 pre-osteoblast cells and increased proliferation which could be attributed to presence of collagen, sGAGs and glycoproteins present in decellularized samples. These biomolecules have been found to promote early adhesion and even to contribute to differentiation. ${ }^{23}$

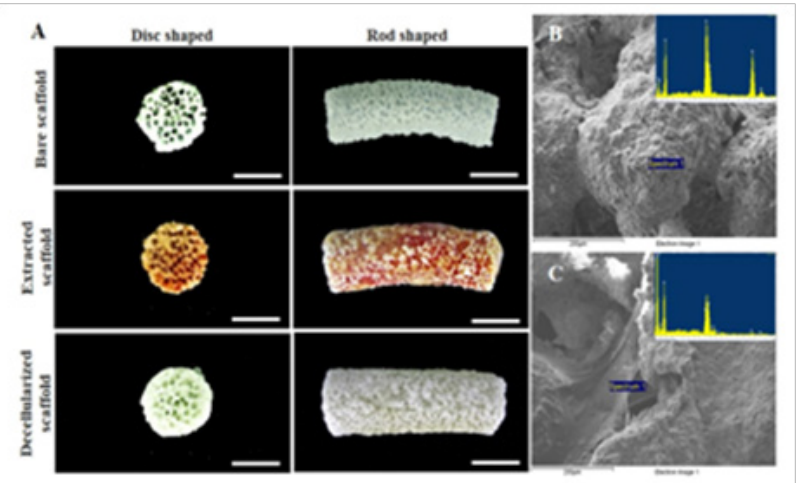

Figure I Images of bare, extracted, and decellularized scaffolds in the form of 5-mm discs for in vitro study and rod shaped for in-vivo study. Reproduced from Ventura et al. ${ }^{15}$ 

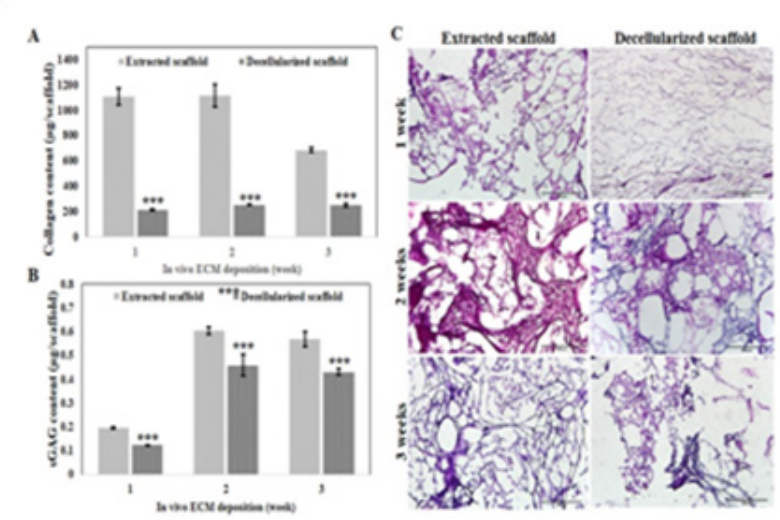

Figure 2 Evaluation of ECM content before and after decellularization. Collagen quantification

\section{A. Sulfated glycosaminoglycan (sGAG) quantification}

B. and periodic acid-Schiff staining for the presence of glycoproteins determination

\section{Reproduced from Ventura et al. ${ }^{15}$}

Based on the characterization of decellularized scaffolds, 2week in-vivo deposited ECM (D2) were chosen for in-vivo studies because it has an even distribution of ECM, a minimum pore size $(75-150 \mu \mathrm{m})$ for bone regeneration, highest compressive strength $(2.05 \pm 0.78 \mathrm{MPa})$, highest content of collagen $(253.98 \pm 3.18 \mu \mathrm{g} / \mathrm{scaffold})$ and sGAGs $(5.92 \pm 0.94 \mu \mathrm{g} / \mathrm{scaffold})$ among the decellularized samples. Bone regeneration potential and biocompatibility of an in vivo deposited ECM in HA scaffolds were evaluated by implanting D2 scaffold in $11 \mathrm{~mm}$ critical radial defect of rabbit for 4 and 8 weeks with bare HA scaffold as a control. Based on results, no hosts' immune reaction was observed after 4 and 8 weeks of in-vivo implantation period due to negligible amount of DNA $(2.06 \pm 0.09 \mu \mathrm{g} / \mathrm{scaffold})$ and RNA $(0.001 \pm 0.002 \mu \mathrm{g} / \mathrm{scaffold})$ content present in decellularized scaffold. Although statistical analysis of the micro CT data showed no significant differences between the bare and decellularized scaffolds, the stained tissue sections showed bare implants developed new bone localized along the implant interface while decellularized HA scaffold showed extensive network of bone/cartilage tissue complex that extends from both ends of the implant interface towards the central region. In addition decellularized groups exhibited enhanced bone fixation of implanted material with the host bone compared with bare groups after 8weeks of in-vivo implantation (Figure 3).

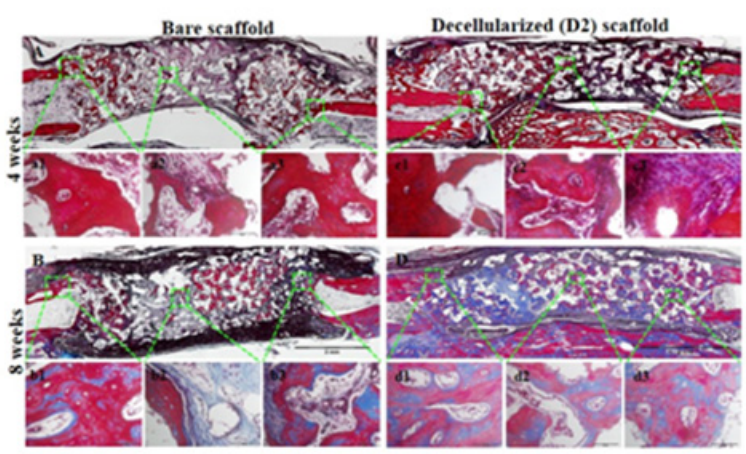

Figure 3 Masson's Trichrome-stained sections of bare $(A, B)$ and decellularized scaffolds(C, D) implanted for 4 and 8 weeks. Scale bar indicates $2 \mathrm{~mm}$ and $50 \mathrm{~mm}$. Reproduced from Ventura et al. ${ }^{15}$

\section{Conclusion}

In summary, this study showed the feasibility of incorporating invivo deposited ECM on hydroxyapatite scaffolds with the use of an animal as a bioreactor. In-vivo deposited ECM in HA scaffolds are cheaper and faster compared with conventional in-vitro or cell derived method. Thorough removal of cellular materials while maintaining functional proteins present in the in-vivo deposited ECM provided an osteoconductive and osteoinductive scaffolds suitable for bone regeneration applications. Decellularized in-vivo deposited ECM on HA scaffolds provides physical support for in-vitro cell attachment and proliferation and enhanced bone regeneration and could be a potential scaffold in bone regeneration applications.

\section{Acknowledgements}

This article was supported by the Basic Science Research Program through the National Research Foundation of Korea (NRF) funded by the Ministry of Education (2015R1A6A1A03032522). Authors would like to thank Mr. Shin-Woo Kim for his assistance of in-vivo operations.

\section{Conflict of interest}

The author declares no conflict of interest.

\section{References}

1. Sano MB, Neal RE 2nd, Garcia PA, et al. Towards the creation of decellularized organ constructs using irreversible electroporation and active mechanical perfusion. Biomed Eng Online. 2010;9:83.

2. Vaccaro AR. The role of the osteoconductive scaffold in synthetic bone graft. Orthopedics. 2002;25(5):S571-S578.

3. Xing Q, Yates K, Tahtinen M, et al. Decellularization of fibroblast cell sheets for natural extracellular matrix scaffold preparation. Tissue Eng Part C Methods. 2015;21(1):77-87.

4. Frantz C, Stewart KM, Weaver VM. The extracellular matrix at a glance. J Cell Sci. 2010;123(Pt 24):4195-200.

5. Järveläinen H, Sainio A, Koulu M, et al. Extracellular matrix molecules: potential targets in pharmacotherapy. Pharmacol Rev. 2009;61(2):198223.

6. Schaefer L, Schaefer RM. Proteoglycans: from structural compounds to signaling molecules. Cell Tissue Res. 2010;339(1):237-246.

7. Gilbert TW, Sellaro TL, Badylak SF. Decellularization of tissues and organs. Biomaterials. 2006;27(19):3675-3683.

8. Hoshiba T, Lu H, Kawazoe N, et al. Decellularized matrices for tissue engineering. Expert Opin Biol Ther. 2010;10(12):1717-1728.

9. Anisimova NY, Kiselevsky MV, Sukhorukova IV, et al. Fabrication method, structure, mechanical, and biological properties of decellularized extracellular matrix for replacement of wide bone tissue defects. $J$ Mech Behav Biomed Mater. 2015;49:255-268.

10. Tabuchi M, Negishi J, Yamashita A, et al. Effect of decellularized tissue powders on a rat model of acute myocardial infarction. Mater Sci Eng C Mater Biol Appl. 2015;56:494-500.

11. Lu H, Hoshiba T, Kawazoe N, et al. Cultured cell-derived extracellular matrix scaffolds for tissue engineering. Biomaterials. 2011;32(36):96589666.

12. Kim IG, Hwang MP1, Du P2, et al. Bioactive cell-derived matrices combined with polymer mesh scaffold for osteogenesis and bone healing. Biomaterials. 2015;50:75-86. 
13. Kulig KM, Luo X, Finkelstein EB, et al. Biologic properties of surgical scaffold materials derived from dermal ECM. Biomaterials. 2013;34(23):5776-5784.

14. Fitzpatrick LE, McDevitt TC. Cell-derived matrices for tissue engineering and regenerative medicine applications. Biomater Sci. 2015;3(1):12-24.

15. Ventura RD, Padalhin AR, Min YK, et al. Bone regeneration using Hydroxyapatite sponge scaffolds with in-vivo deposited extracellular matrix (ECM). Tissue Eng Part A. 2015;21(21-22):2649-2661.

16. Crapo PM, Gilbert TW, Badylak SF. An overview of tissue and whole organ decellularization processes. Biomaterials. 2011;32(12):32333243 .

17. Nagata S, Hanayama R, Kawane K. Autoimmunity and the clearance of dead cells. Cell. 2010;140(5):619-630.

18. Manfredi AA, Capobianco A, Bianchi ME, et al. Regulation of dendriticand T-cell fate by injury-associated endogenous signals. Crit Rev Immunol. 2009;29(1):69-86.
19. Brown SA, Lemons JE, Mateo NB. New Products and Standards. In Biomaterials Science. In: Ratner BD, Lemons ASHJSE, editors. USA: Academic Press; 1996. p. 457-464.

20. Zhang Q, Raoof M, Chen Y, et al. Circulating mitochondrial DAMPs cause inflammatory responses to injury. Nature. 2010;464(7285):104 107.

21. Rosso F, Giordano A, Barbarisi M, et al. From cell-ECM interactions to tissue engineering. J Cell Physiol. 2004;199(2):174-180.

22. Rozario T, DeSimone DW. The extracellular matrix in development and morphogenesis: a dynamic view. Dev Biol. 2010;341(1):126-140.

23. Cortiella J, Niles J, Cantu A, et al. Influence of acellular natural lung matrix on murine embryonic stem cell differentiation and tissue formation. Tissue Eng Part A. 2010;16(8):2565-2580. 